\title{
Endogenous Factors Causative of Spontaneous DNA Damage that Leads to Random Integration in Human Cells
}

\author{
Haruna Kamekawa1 ${ }^{1}$, Aya Kurosawa ${ }^{1}$, Masumi Umehara ${ }^{1}$, Eriko Toyoda ${ }^{1}$ and Noritaka Adachi ${ }^{1,2 *}$ \\ ${ }^{1}$ Graduate School of Nanobioscience, Yokohama City University, Yokohama 236-0027, Japan \\ ${ }^{2}$ Advanced Medical Research Center, Yokohama City University, Yokohama 236-0004, Japan
}

\begin{abstract}
Random integration is a phenomenon in which transfected DNA molecules integrate into (random sites of) the host genome via non-homologous recombination. Although it is assumed that repair of DNA double-strand breaks leads to random integration events, how these endogenous DNA lesions are generated in living cells is poorly understood. In this study, we present evidence that DNA topoisomerase Il $($ Top2 $\alpha$ ) and reactive oxygen species (ROS) are responsible for causing genomic DNA damage that leads to random integration. Specifically, we employed a human pre-B lymphocyte cell line to examine the effects of cellular Top2 expression levels and oxygen concentrations during cell culture. We find that treating cells with Top2 $\alpha$ siRNA significantly reduces random integration frequency, while the absence of Top2 $\beta$ had little or no impact. We also show that cells continuously cultured under low (3\%) oxygen culture conditions after electroporation display reduced random integration frequency compared to that under normal $(21 \%)$ oxygen conditions. These findings support the notion that Top2 $\alpha$ protein and ROS are endogenous factors that can produce DNA damage leading to random integration of transfected DNA in human cells.
\end{abstract}

Keywords: DNA damage; Random integration; Top $2 \alpha$; Top $2 \beta$

\section{Introduction}

Mammalian cells possess the ability to perform nonhomologous recombination reactions, which require little or no sequence homology between DNA substrates [1,2]. Recent evidence indicates that the nonhomologous end-joining (NHEJ) pathway accounts for most, but not all, of nonhomologous recombination reactions occurring in the cell nucleus [3]. NHEJ and homologous recombination are the two major pathways for repairing DNA double-strand breaks (DSBs) that result from endogenous mechanisms as well as exposure to exogenous genotoxic agents [4]. A practical application of nonhomologous recombination is the generation of transfectants (i.e., random integrants) that stably express a transgene(s) of interest. Although the precise mechanism of random integration is not fully understood, it is believed that random integration results from non-homologous recombination-mediated repair, particularly NHEJ, of a spontaneous chromosomal DSB accidentally induced by endogenous factors [5-7]. In this study, we focused on DNA topoisomerase II (Top2) and reactive oxygen species (ROS) as the endogenous factors that induce DSBs causative of random integration.

Top2 is a ubiquitous nuclear enzyme that alters the topological structure of DNA and chromosomes through a transient DSB and subsequent religation of the DSB [8]. The enzyme has been implicated in many aspects of DNA metabolism, including DNA replication, transcription, and chromosome condensation/segregation [8]. Mammalian cells express two genetically distinct Top2 isoforms, $\alpha$ and $\beta$, which are differentially regulated and play different roles in living cells [8]. Top2 $\alpha$ is most abundantly expressed in rapidly growing tissues and its expression is cell cycle-regulated, peaking in $\mathrm{G}_{2} / \mathrm{M}$, whereas the $\beta$-isoform is expressed in virtually all tissues and throughout the cell cycle [9-13]. Top2-targeting agents, such as etoposide, are among the most effective and widely used anticancer drugs in cancer chemotherapy [14]. These agents are referred to as "Top2 poisons", as they convert the essential enzyme into a highly cytotoxic DNA-damaging agent through the formation of "cleavage complex" (also called "cleavable complex"), in which a Top2-linked DNA strand-passing intermediate is stabilized, allowing the generation of a DSB [14]. It has been demonstrated that NHEJ plays a crucial role in the repair of Top2 inhibitor-induced DSBs $[15,16]$. Earlier work has shown that Top2 inhibitors can enhance random integration via nonhomologous recombination in mammalian cells $[17,18]$. Intriguingly, we have recently shown that inhibition of Top $2 \alpha$, not Top $2 \beta$, is critical for the enhancement of random integration [19]. It remains to be elucidated, however, whether Top $2 \alpha$ protein is actually involved in spontaneously occurring (i.e., Top2 inhibitor-independent) random integration events under physiological conditions, namely in the absence of forced poisoning of Top2 protein.

ROS are produced naturally as a product of oxidative metabolism in the mitochondrial electron transport chain and include superoxide anion radical, hydrogen peroxide and the hydroxyl free radical [20]. ROS are capable of inducing oxidative damage of DNA, including single-strand breaks and base and nucleotide modifications, such as 8-oxo-2'-deoxyguanine [21]. Intriguingly, ROS are also reported to generate chromosomal DSBs and that low oxygen culture conditions can reduce these DNA lesions [22]. We therefore reasoned that random integration frequency might be decreased if the transfected cells were cultured under low oxygen conditions.

In this report, we find using the human cell line Nalm-6 that treating cells with Top $2 \alpha$ siRNA significantly reduces random integration frequency, thus providing direct evidence that spontaneous Top $2 \alpha$-induced DSBs indeed cause random integration in human cells. We also show that cells continuously cultured at $3 \%$ oxygen concentration $\left(3 \% \mathrm{O}_{2} / 5 \% \mathrm{CO}_{2} / 92 \% \mathrm{~N}_{2}\right)$ display reduced random integration frequency compared to that at $21 \%$ oxygen concentration, providing the first direct evidence that random integration is decreased under low oxygen culture conditions. From these results, we propose that Top $2 \alpha$ protein and ROS are endogenous factors that can produce

*Corresponding author: Noritaka Adachi, Graduate School of Nanobioscience Yokohama City University, Yokohama 236-0027, Japan, Tel: 81-45-787-2228; Fax 81-45-787-2228; E-mail: nadachi@yokohama-cu.ac.jp

Received August 29, 2013; Accepted October 01, 2013; Published October 03 2013

Citation: Kamekawa H, Kurosawa A, Umehara M, Toyoda E, Adachi N (2013) Endogenous Factors Causative of Spontaneous DNA Damage that Leads to Random Integration in Human Cells. Gene Technology 2: 105. doi: 10.4172/23296682.1000105

Copyright: (c) 2013 Kamekawa H, et al. This is an open-access article distributed under the terms of the Creative Commons Attribution License, which permits unrestricted use, distribution, and reproduction in any medium, provided the original author and source are credited. 
Citation: Kamekawa H, Kurosawa A, Umehara M, Toyoda E, Adachi N (2013) Endogenous Factors Causative of Spontaneous DNA Damage that Leads to Random Integration in Human Cells. Gene Technology 2: 105. doi: 10.4172/2329-6682.1000105

Page 2 of 5

DNA damage leading to random integration of transfected DNA in human cells.

\section{Materials and Methods}

\section{Cells and culture conditions}

The human pre-B cell line Nalm- 6 and its derivatives were cultured in ES medium (Nissui Seiyaku, Tokyo, Japan) supplemented with $10 \%$ calf serum (Hyclone, Logan, UT) and $50 \mu \mathrm{M} 2$-mercaptoethanol at $37^{\circ} \mathrm{C}$ in a humidified atmosphere containing $5 \% \mathrm{CO}_{2}$ [23]. TOP $2 A^{+/-}$ and $\mathrm{TOP}_{2} \mathrm{~B}^{-1}$ cells were created by gene targeting, as reported previously [24] (Figure S1). A TOP2B- ${ }^{-/} \mathrm{TOP} 2 \mathrm{~A}^{+/-}$cell line was created by gene targeting (heterozygous disruption) of the TOP $2 A$ gene using the TOP $2 B^{-/}$cells, as described previously (Figure S1).

\section{Drugs}

Etoposide was purchased from BioVision (100 mM;Mountain View, CA) and dissolved in dimethyl sulfoxide. Bleomycin was purchased from Wako (Osaka, Japan) and dissolved in distilled water to a concentration of $1 \mathrm{mg} / \mathrm{ml}$. These drugs were stored frozen in aliquots at $-20^{\circ} \mathrm{C}$.

\section{Transfection and integration assays}

Random integration assays were carried out using Nalm- 6 cells, essentially as described $[7,19]$. Briefly, $4 \times 10^{6}$ cells were electroporated with $4 \mu \mathrm{g}$ of NotI-linearized pLucPuro per $40-\mu \mathrm{l}$ cuvette of Electro Gene Transfer Equipment (GTE-1; Shimadzu, Kyoto, Japan). After 15 min, cells were transferred into growth medium with or without drugs, and cultured for $24 \mathrm{hr}$ at $21 \%$ or $3 \%$ oxygen concentration. Transfected cells were then collected, counted and plated into agarose medium containing $0.5 \mu \mathrm{g} / \mathrm{ml}$ puromycin (Wako). Meanwhile, small aliquots of transfected cells were plated into drug-free agarose medium to determine the plating efficiency. After two-week cultivation, the integration frequency was calculated by dividing the number of puromycin-resistant colonies by that of cells plated multiplied by the plating efficiency. pLucPuro was constructed by subcloning a SalI fragment containing a PGK promoter, a puromycin-resistance gene and polyA signal sequences from pPGKPuro into SalI-digested pGL413 (Figure S2).

\section{Small interfering RNA (siRNA)}

Top2 $\alpha$-targeting siRNA (sense, 5 '-AAAAGACUGUCUGUUGAAAGAdTdT-3'; antisense,5'-UCUUUCAACAGACAGUCUUUUdTdT-3') corresponding to nucleotides 76 to 96 relative to the first nucleotide of the start codon was purchased from Qiagen (Tokyo, Japan) [24]. The siRNA was diluted with RNase-free water and stored frozen at $-20^{\circ} \mathrm{C}$. Log-phase cells were electroporated with 200 pmol of siRNA alone or along with NotI-linearized pLuc Puro, followed by western blot analysis or random integration assays.

\section{Luciferase assays}

Luciferase assays were performed as described previously [19]. Briefly, after electroporation with pLucPuro, cells were cultured in growth medium for $5 \mathrm{hr}$. An aliquot $\left(3 \times 10^{4}\right.$ cells $)$ was then suspended in $50 \mu \mathrm{l}$ of growth medium, and subjected to luciferase assays using the ONE-Glo Luciferase Assay System (Promega, WI) according to the manufacturer's instructions.

\section{Western blot analysis}

Cells were suspended in lysis buffer (20 mM Tris- $\mathrm{HCl}(\mathrm{pH} 8.0)$,
0.2 mM EDTA (pH 8.0), 10\% glycerol, 2 mM DTT, $150 \mathrm{mM} \mathrm{KCl,} \mathrm{and}$ protease inhibitor cocktails (Sigma-Aldrich, St Louis, MO)), sonicated three times for $5 \mathrm{sec}$, and centrifuged at $15,000 \mathrm{rpm}$ for $30 \mathrm{~min}$ at $4^{\circ} \mathrm{C}$. Lysates corresponding to $20 \mu \mathrm{g}$ were mixed with $2 \mathrm{xSDS}$ sample buffer, and heated at $95^{\circ} \mathrm{C}$ for $2 \mathrm{~min}$, and subjected to electrophoresis on a $0.1 \%$ SDS- $6 \%$ polyacrylamide gel. Polypeptides were transferred onto a PVDF membrane in a semidry-type blotting apparatus for 1 $\mathrm{hr}$ at $100 \mathrm{~mA}$. Molecular masses of polypeptides were estimated by comparison with migration of Precision plus protein all blue standards (Bio-Rad, Hercules, CA). Membranes were first blocked with 5\% nonfat milk powder in Tris-buffered saline containing 0.05\% Tween 20 . The antibodies used were mouse monoclonal antibody against human Top $2 \alpha$ or Top $2 \beta$, mouse anti-human Ku70 monoclonal antibody (1:500; BD Transduction Laboratories, Bedford, MA) and mouse antihuman actin monoclonal antibody (1:2000; Sigma-Aldrich). Levels of expression were quantified using a Fuji Image analyzer LAS-1000UV mini and a MultiGauge software (Fuji Film, Tokyo, Japan) [24].

\section{Results}

\section{Top2a siRNA-treated cells exhibit reduced random integration frequencies}

As mammalian cells express two genetically distinct isoforms of Top2, we first analyzed the contribution of each isoform to random integration. Specifically, we examined the random integration frequency in Nalm-6 wild-type cells (proficient for Top $2 \alpha$ and Top $2 \beta$ ), TOP $2 A^{+/-}$ (heterozygously null for Top $2 \alpha$ ) and $\mathrm{TOP}_{2} \mathrm{~B}^{-/}$cells (homozygously null for Top2 $\beta$ ) (Figure S1). As shown in Figure 1A, the random integration frequency of pLucPuro plasmid in these cells was comparable to that in wild-type cells. Transient expression analysis revealed no significant change in luciferase activity (Figure 1B). These data suggest that neither heterozygous disruption of TOP $2 A$ nor homozygous disruption of TOP2B has little or no effect on the frequency of random integration. We further examined the contribution of Top2 $\alpha$ by constructing a TOP $2 B^{--} \mathrm{TOP}_{2} \mathrm{~A}^{+/-}$cell line by gene targeting (Figure $\mathrm{S} 1$ ), which led us find that the heterozygous disruption of TOP $2 A$ does not affect the random integration frequency even in the complete absence of Top $2 \beta$ (Figure 1A)

As the human TOP2A gene is essential for cell proliferation $[25,26]$, we next employed Top $2 \alpha$ siRNA to analyze the random integration frequency in cells with greatly reduced Top $2 \alpha$ levels. Western blot
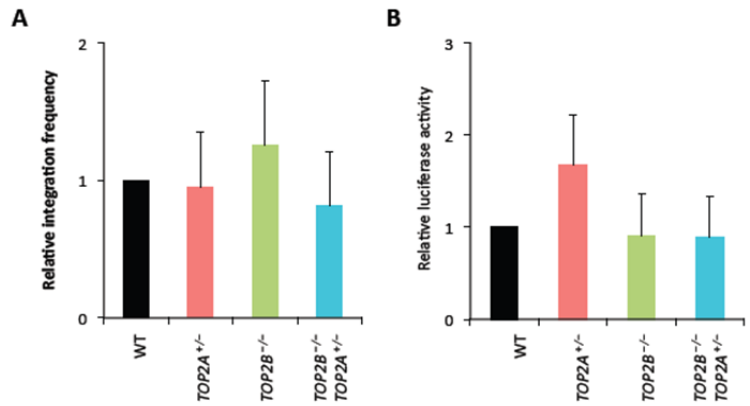

Figure 1: TOP2 mutants retain wild-type levels of random integration frequency. (A, B) Wild-type cells and TOP2 mutants were electroporated with pLucPuro and subjected to random integration assay $(A)$ or luciferase assay (B). The random integration frequency and the luciferase activity in wildtype cells were taken as 1 , and the relative integration frequency or luciferase activity in TOP2 mutants was calculated. Data are the mean \pm S.D. of at least three independent experiments. 
Citation: Kamekawa H, Kurosawa A, Umehara M, Toyoda E, Adachi N (2013) Endogenous Factors Causative of Spontaneous DNA Damage that Leads to Random Integration in Human Cells. Gene Technology 2: 105. doi: 10.4172/2329-6682.1000105

Page 3 of 5

analysis showed that Top $2 \alpha$ expression level in Top $2 \alpha$ siRNA-treated cells was reduced $\sim 90 \%$ compared to mock-treated cells (Figure $2 \mathrm{~A}$ and $2 \mathrm{~B}$ ). As shown in Figure 2C, the random integration frequency in Top $2 \alpha$-knockdown cells was reduced $\sim 40 \%$. Luciferase activity in Top2a-knockdown cells was comparable to that in mock-treated cells (Figure 2D). These results suggest that random integration frequency is reduced when Top $2 \alpha$ expression is strongly suppressed. This is in sharp contrast to our previous finding that absence of Top $2 \beta$ had little or no effect on the frequency of random integration [19].

\section{Random-integration enhancement by Top2 inhibitor is Top2a dependent}

We next examined the effect of DSBs induced by the Top2 inhibitor etoposide on random integration frequency. Etoposide targets both isoforms of Top2 in a cell [14]. As shown in Figure 3A, random integration frequency was increased in all the cell lines in a drug concentration-dependent manner. Importantly, the level of enhancement was less prominent in $\mathrm{TOP} 2 \mathrm{~A}^{+/-}$and $\mathrm{TOP} 2 \mathrm{~B}^{-/-} \mathrm{TOP} 2 \mathrm{~A}^{+/-}$ cells. In contrast, random integration frequency in $T O P 2 B^{-/}$cells was comparable to that observed in wild-type cells. Very similar results were obtained using NK314, a Top2 $\alpha$-specific poison [24] (data not shown). These results suggest that the repair process for Top $2 \alpha$-mediated, not Top $2 \beta$-mediated, DSBs is involved in Top 2 poison-induced random integration.

We also examined the contribution of Top2 expression levels to bleomycin-enhanced random integration. Bleomycin binds transition metals, $\mathrm{Fe}(\mathrm{II})$ or $\mathrm{Cu}(\mathrm{I})$, and oxygen and, in the presence of a oneelectron reductant, can catalyze formation of single-stranded and double-stranded DNA lesions [27]. We have previously shown that, similar to Top2 inhibitors, bleomycin can enhance random integration in human somatic cells [28]. As expected, bleomycin treatment of cells greatly enhanced random integration (Figure $3 \mathrm{~B}$ ) and, unlike the case

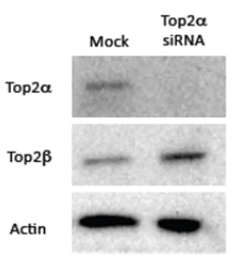

C

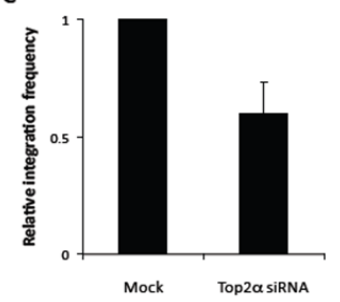

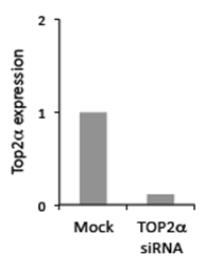

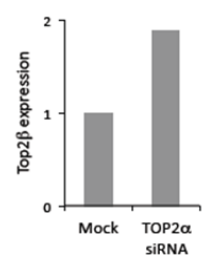

D

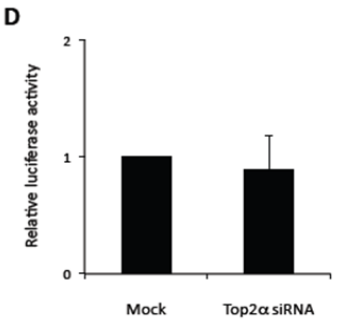

Figure 2: The random integration frequency in Top2 $\alpha$ siRNA-treated cells is reduced. $(A, B)$ Western blot analysis for Top2 in Top2 $\alpha$ siRNAtreated cells. Actin served as a loading control. (B) Relative expression levels of Top2 proteins in Top2 $\alpha$ siRNA-treated cells. $(C, D)$ Mock-treated cells and Top2 $\alpha$ siRNA-treated cells were electroporated with linearized pLucPuro and subjected to random integration assay (C) or luciferase assay (D). The random integration frequency or the luciferase activity in mock-treated cells was taken as 1 , and the relative integration frequency or luciferase activity in Top2 $\alpha$ siRNA-treated cells was calculated. Data are the mean \pm S.D. of three independent experiments. of etoposide, the level of enhance mention $\mathrm{TOP}^{2} \mathrm{~A}^{+-}$and $\mathrm{TOP} 2 \mathrm{~B}^{-1}$ TOP $2 A^{+/-}$cells was comparable to that in wild-type cells. These results suggest that Top $2 \alpha$ does not participate in bleomycin-enhanced random integration, while it is specifically involved in Top2 poisoninduced random integration.

\section{Low oxygen culture conditions reduce the frequency of random integration}

The above data revealed that Top $2 \alpha$ participates in spontaneously occurring random integration as well as Top2 inhibitor-induced random integration. Apparently, however, other endogenous factors should also be involved in causing DNA damage that leads to random integration events. Thus, we next focused on endogenous free radicals, ROS, as the factor that induces spontaneous chromosome breaks. For this purpose, we cultured transfected cells for $24 \mathrm{hr}$ under normal (21\%) or low (3\%) oxygen conditions, and performed colony formation under normal or low oxygen conditions. More specifically, we examined the random integration frequency by culturing cells under either of the following culture conditions (Figure 4A): (i) cells were cultured
A

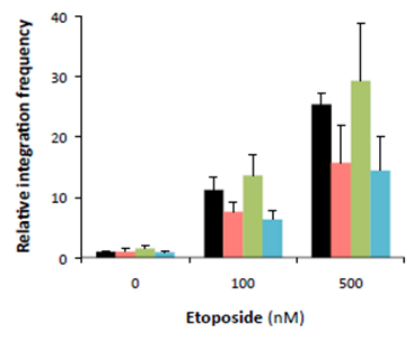

B

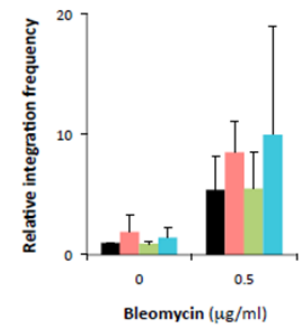

Figure 3: Random-integration enhancement by etoposide is less prominent in TOP2A $A^{+/}$cells. $(A, B)$ Wild-type cells and TOP2 mutants were electroporated with linearized pLucPuro, and treated with the indicated concentrations of etoposide (A) or bleomycin (B) for $24 \mathrm{hr}$. The random integration frequency in wild-type cells was taken as 1 , and the relative integration frequency in TOP2 mutants was calculated. Data are the mean \pm S.D. of three independent experiments. Symbols are as in Figure 1.

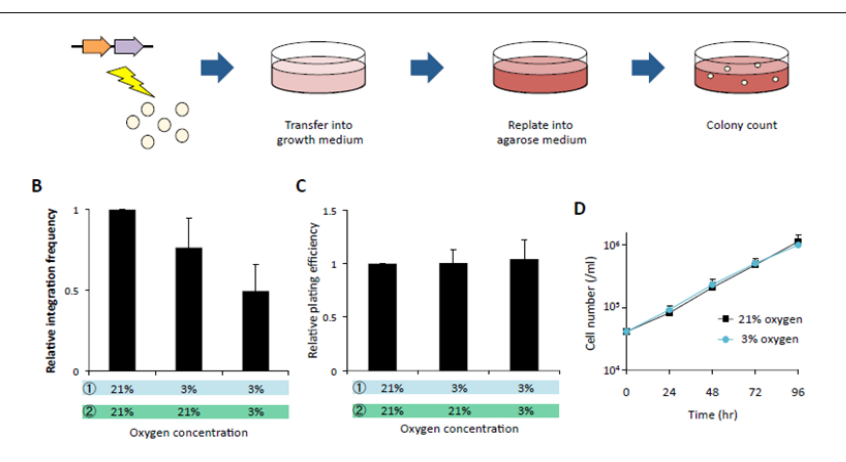

Figure 4: The $3 \%$ oxygen condition reduces the frequency of random integration. (A) Strategy of random integration assay. Nalm-6 cells $\left(4 \times 10^{6}\right)$ were electroporated with $4 \mu \mathrm{g}$ of linearized plasmid vector and cultured for 24 hr at $21 \%$ or $3 \%$ oxygen concentration, and then allowed for colony formation at $21 \%$ or $3 \%$ oxygen concentration. (B) Relative integration frequency at the indicated oxygen conditions. Cells were electroporated with linearized pLucPuro, and incubated at the indicated oxygen concentration. Data are the mean \pm S.D. of three independent experiments. (C) Plating efficiencies of cells cultured under the indicated oxygen conditions after electroporation. Data are the mean \pm S.D. of three independent experiments. (D) Growth curves of cells cultured at $21 \%$ or $3 \%$ oxygen concentration. Data are the mean \pm S.D. of three independent experiments. Where absent, error bars fall within symbols. 
continuously under normal oxygen conditions after electroporation, (ii) cells were cultured under low oxygen conditions during cultivation after electroporation, and then allowed for colony formation under normal oxygen conditions, or (iii) cells were continuously cultured under low oxygen conditions after electroporation.

As shown in Figure 4B, we found that low oxygen concentration did reduce the frequency of random integration. In particular, random integration frequency was decreased by half in cells continuously cultured under low oxygen conditions after electroporation. It should be noted that the plating efficiency and the growth rate of cells under low oxygen conditions were indistinguishable from that under normal oxygen conditions, indicating that low oxygen culture conditions do not disturb cell proliferation per se (Figure 4C and 4D).

\section{Discussion}

It has been generally assumed that random integration of foreign DNA results from the repair of DNA damage, specifically spontaneous chromosomal DSBs that are caused by endogenous factors. However, it is largely unknown how these DSBs are generated in the cell nucleus. In this study, we focused on cellular Top2 proteins ( $\alpha$ and $\beta$ ) and free radicals as the factors responsible for endogenous DSBs, and examined their contribution to random integration frequency. The data presented here clearly indicate that Top $2 \alpha$ participates in spontaneously occurring random integration as well as Top2 inhibitorinduced random integration. Similarly importantly, our data provide the first evidence that oxygen concentration during cell culture does affect the frequency of random integration in human cells.

Our observation that Top $2 \alpha$ siRNA-treated cells display reduced random integration frequency provides evidence that spontaneously occurring Top $2 \alpha$-mediated DSBs can cause random integration. We also found that inhibition of Top $2 \alpha$ not Top $2 \beta$, is important for etoposide-induced random integration. Why are Top $2 \alpha$-mediated DSBs specifically involved in random integration? Earlier work has identified mammalian cell lines that do not express Top2 $\beta$, and indeed Top2 $\beta$-null cells do not exhibit growth defects at the cellular level [24,2932]. Thus, it seems quite reasonable that only Top $2 \alpha$ is responsible for causing random integration, while Top $2 \beta$ is not. However, we reported previously that Nalm- 6 cells express similar levels of Top $2 \alpha$ and Top $2 \beta$ [33], which does not account for the Top $2 \alpha$ dominance in causing random integration. One possibility might be a cell cycle dependency of Top $2 \alpha$-mediated DSBs. Top $2 \alpha$ expression is cell cycle-regulated, peaking in $\mathrm{G}_{2} / \mathrm{M}$, whereas the $\beta$-isoform is expressed throughout the cell cycle [9-11]. Possibly, Top2 $\alpha$-mediated DSBs that arise in $G_{2} / M$ phase might be convenient for random integration to occur. Interestingly, Cowell et al. [34] have recently reported that $T_{O P} 2 B^{-/}$Nalm-6 cells have roughly equal amounts of chromosomal DSBs after etoposide treatment when compared to wild-type Nalm-6 cells. This finding clearly indicates that Top $2 \beta$ does not play a major role in producing etoposide-induced DSBs, a notion that well explains our observations described herein. It should be noted, however, that Top2 $\beta$-mediated DSBs, albeit fewer in number, are actually induced by etoposide treatment, and this type of DNA damage governs the genotoxicity of drug-treated cells [34]. Unlike Top $2 \alpha$, Top $2 \beta$ is thought to be specifically required for transcription, and thus binds to promoter regions in the genome $[35,36]$. Possibly, Top $2 \beta$-mediated DSBs, if any, might be strictly regulated not to use NHEJ for repair, in a manner that does not permit genomic incorporation of foreign DNA. This idea may well account for the inertness of Top $2 \beta$ in causing random integration.

We have also shown in this study that culturing cells under low oxygen conditions results in a reduced random integration frequency.
It is assumed that low oxygen culture conditions should reduce cellular free radicals, resulting in a decrease in the amount of spontaneous DNA damage (strand breaks as well as oxidative damage) in the genome. Indeed, Karanjawala et al. [22] have reported that the number of spontaneous DSBs due to ROS produced within a cell is substantial and that oxygen metabolism actually causes chromosomal DSBs that likely rely on NHEJ for repair. More specifically, cells lacking DNA ligase IV (the critical DNA ligase for NHEJ to be completed) had increased levels of ROS [22]. Karanjawala et al. [22] further showed that low oxygen $(3 \%)$ culture conditions could reduce those DSBs. These findings are consistent with our observation that the random integration frequency decreases in cells cultured under low oxygen conditions, due to a reduction of ROS induced-DSBs in the cell. It should be noted that the fact that the low oxygen culture condition is effective in reducing genomic DNA damage as well as unwanted random integration of foreign DNA might have implications for future gene/cell therapy. For example, iPS cells have been shown to be more stably maintained under low oxygen culture conditions ([37] and our unpublished observations). Even more importantly, as random integration is a major obstacle in gene targeting via homologous recombination, a decreased random integration frequency should be preferable to increasing the frequency of targeted gene inactivation/ correction [7]. It is therefore expected that artificial manipulation of random integration will contribute to significant improvements in gene-targeting technology.

\section{References}

1. Roth DB, Wilson JH (1985) Relative rates of homologous and nonhomologous recombination in transfected DNA. Proc Natl Acad Sci U S A 82: 3355-3359.

2. Roth DB, Wilson JH (1988) Illegitimate recombination in mammalian cells American Society for Microbiology. 621-653

3. Moore JK, Haber JE (1996) Cell cycle and genetic requirements of two pathways of nonhomologous end-joining repair of double-strand breaks in Saccharomyces cerevisiae. Mol Cell Biol 16: 2164-2173.

4. Kass EM, Jasin M (2010) Collaboration and competition between DNA doublestrand break repair pathways. FEBS Lett 584: 3703-3708.

5. Fattah FJ, Lichter NF, Fattah KR, Oh S, Hendrickson EA (2008) Ku70, an essential gene, modulates the frequency of rAAV-mediated gene targeting in human somatic cells. Proc Natl Acad Sci U S A 105: 8703-8708.

6. Bertolini LR, Bertolini M, Maga EA, Madden KR, Murray JD (2009) Increased gene targeting in $\mathrm{Ku} 70$ and $\mathrm{Xrcc} 4$ transiently deficient human somatic cells. Mol Biotechnol 41: 106-114.

7. liizumi S, Kurosawa A, So S, Ishii Y, Chikaraishi Y, et al. (2008) Impact of nonhomologous end-joining deficiency on random and targeted DNA integration: implications for gene targeting. Nucleic Acids Res 36: 6333-6342.

8. Nitiss JL (2009) DNA topoisomerase II and its growing repertoire of biologica functions. Nat Rev Cancer 9: 327-337.

9. Adachi N, Nomoto M, Kohno K, Koyama H (2000) Cell-cycle regulation of the DNA topoisomerase llalpha promoter is mediated by proximal CCAAT boxes: possible involvement of acetylation. Gene 245: 49-57.

10. Woessner RD, Mattern MR, Mirabelli CK, Johnson RK, Drake FH (1991) Proliferation- and cell cycle-dependent differences in expression of the 170 kilodalton and 180 kilodalton forms of topoisomerase II in NIH-3T3 cells. Cell Growth Differ 2: 209-214.

11. Padget K, Pearson AD, Austin CA (2000) Quantitation of DNA topoisomerase Ilalpha and beta in human leukaemia cells by immunoblotting. Leukemia 14: 1997-2005.

12. Rattner JB, Hendzel MJ, Furbee CS, Muller MT, Bazett-Jones DP (1996) Topoisomerase II alpha is associated with the mammalian centromere in a cell cycle- and species-specific manner and is required for proper centromere/ kinetochore structure. J Cell Biol 134: 1097-1107.

13. Hsiang YH, Wu HY, Liu LF (1988) Proliferation-dependent regulation of DNA topoisomerase II in cultured human cells. Cancer Res 48: 3230-3235. 
Citation: Kamekawa H, Kurosawa A, Umehara M, Toyoda E, Adachi N (2013) Endogenous Factors Causative of Spontaneous DNA Damage that Leads to Random Integration in Human Cells. Gene Technology 2: 105. doi: 10.4172/2329-6682.1000105

14. Nitiss JL (2009) Targeting DNA topoisomerase II in cancer chemotherapy. Nat Rev Cancer 9: 338-350.

15. Kurosawa A, Saito S, So S, Hashimoto M, Iwabuchi K, et al. (2013) DNA Ligase IV and Artemis Act Cooperatively to Suppress Homologous Recombination in Human Cells: Implications for DNA Double-Strand Break Repair. PLoS One 8 : e72253.

16. Adachi N, Suzuki H, liizumi S, Koyama H (2003) Hypersensitivity of nonhomologous DNA end-joining mutants to VP-16 and ICRF-193: implications for the repair of topoisomerase II-mediated DNA damage. J Biol Chem 278: 35897-35902.

17. Fujimaki K, Aratani Y, Fujisawa S, Motomura S, Okubo T, et al. (1996) DNA topoisomerase II inhibitors enhance random integration of transfected vectors into human chromosomes. Somat Cell Mol Genet 22: 279-290.

18. Aratani Y, Andoh T, Koyama H (1996) Effects of DNA topoisomerase inhibitors on nonhomologous and homologous recombination in mammalian cells. Mutat Res 362: 181-191

19. Toyoda E, Kurosawa A, Kamekawa H, Adachi N (2009) Topoisomerase Ilalpha inhibition following DNA transfection greatly enhances random integration in a human pre-B lymphocyte cell line. Biochem Biophys Res Commun 382: 492 496

20. Sena LA, Chandel NS (2012) Physiological roles of mitochondrial reactive oxygen species. Mol Cell 48: 158-167.

21. Dizdaroglu M (2012) Oxidatively induced DNA damage: mechanisms, repair and disease. Cancer Lett 327: 26-47.

22. Karanjawala ZE, Murphy N, Hinton DR, Hsieh CL, Lieber MR (2002) Oxygen metabolism causes chromosome breaks and is associated with the neuronal apoptosis observed in DNA double-strand break repair mutants. Curr Biol 12 397-402.

23. Adachi N, Kurosawa A, Koyama $H$ (2008) Highly proficient gene targeting by homologous recombination in the human pre-B cell line Nalm-6. Methods Mo Biol 435: 17-29.

24. Toyoda E, Kagaya S, Cowell IG, Kurosawa A, Kamoshita K, et al. (2008) NK314, a topoisomerase II inhibitor that specifically targets the alpha isoform. J Biol Chem 283: 23711-23720.

25. Akimitsu N, Adachi N, Hirai H, Hossain MS, Hamamoto H, et al. (2003) Enforced cytokinesis without complete nuclear division in embryonic cells depleting the activity of DNA topoisomerase Ilalpha. Genes Cells 8: 393-402.

26. Carpenter AJ, Porter AC (2004) Construction, characterization, and complementation of a conditional-lethal DNA topoisomerase Ilalpha mutant human cell line. Mol Biol Cell 15: 5700-5711.

27. Chen J, Stubbe J (2005) Bleomycins: towards better therapeutics. Nat Rev Cancer 5: 102-112

28. Nakayama C, Adachi N, Koyama H (1998) Bleomycin enhances random integration of transfected DNA into a human genome. Mutat Res 409: 1-10.

29. Chen M, Beck WT (1995) DNA topoisomerase II expression, stability, and phosphorylation in two VM-26-resistant human leukemic CEM sublines. Oncol Res 7: 103-111.

30. Dereuddre S, Delaporte C, Jacquemin-Sablon A (1997) Role of topoisomerase II beta in the resistance of 9-OH-ellipticine-resistant Chinese hamster fibroblasts to topoisomerase II inhibitors. Cancer Res 57: 4301-4308.

31. Grue P, Grässer A, Sehested M, Jensen PB, Uhse A, et al. (1998) Essentia mitotic functions of DNA topoisomerase llalpha are not adopted by topoisomerase Ilbeta in human H69 cells. J Biol Chem 273: 33660-33666.

32. Yang X, Li W, Prescott ED, Burden SJ, Wang JC (2000) DNA topoisomerase Ilbeta and neural development. Science 287: 131-134.

33. Adachi N, So S, liizumi S, Nomura Y, Murai K, et al. (2006) The human pre-B cell line Nalm-6 is highly proficient in gene targeting by homologous recombination. DNA Cell Biol 25: 19-24.

34. Cowell IG, Sondka Z, Smith K, Lee KC, Manville CM, et al. (2012) Model for $M L L$ translocations in therapy-related leukemia involving topoisomerase II mediated DNA strand breaks and gene proximity. Proc Natl Acad Sci U S A 109: 8989-8994.

35. Ju BG, Lunyak VV, Perissi V, Garcia-Bassets I, Rose DW, et al. (2006) A topoisomerase Ilbeta-mediated dsDNA break required for regulated transcription. Science 312: 1798-1802.

36. Lis JT, Kraus WL (2006) Promoter cleavage: a topollbeta and PARP-1 collaboration. Cell 125: 1225-1227.

37. Guo CW, Kawakatsu M, Idemitsu M, Urata Y, Goto S, et al. (2013) Culture under low physiological oxygen conditions improves the stemness and quality of induced pluripotent stem cells. J Cell Physiol 228: 2159-2166. 